\title{
MAGNETICALLY RESPONSIVE MATERIALS FOR SOLID PHASE EXTRACTION
}

\author{
Ivo Safarik ${ }^{1,2^{*}}$, Jitka Prochazkova ${ }^{1}$, Eva Baldikova ${ }^{1}$, Kristyna Pospiskova ${ }^{2}$ \\ ${ }^{1}$ Department of Nanobiotechnology, Biology Centre, ISB, CAS, Na Sadkach 7, 37005 Ceske Budejovice, \\ Czech Republic \\ ${ }^{2}$ Regional Centre of Advanced Technologies and Materials, Palacky University, Slechtitelu 27, \\ 78371 Olomouc, Czech Republic \\ *E-mail of corresponding author: ivosaf@yahoo.com
}

\begin{abstract}
Magnetically responsive materials have found many important applications in analytical chemistry. In this short review the basic information about Magnetic solid phase extraction and Magnetic textile solid phase extraction is given. These analytical techniques enable to preconcentrate target biologically active compounds or pollutants from water samples. Both procedures enable to lower the limit of detection using conventional analytical procedures.
\end{abstract}

Keywords: Magnetic solid phase extraction, Magnetic textile solid phase extraction, preconcentration, elution, image analysis

\begin{tabular}{c}
\hline Received: $01.05 .2019 . /$ Accepted: 30.05 .2019$. \\
Published online: 16.07 .2019$. \\
\hline
\end{tabular}

Review paper

\section{INTRODUCTION}

Sensitive analytical techniques have to be used to monitor the concentration of target biologically active compounds, organic and inorganic pollutants or radionuclides present in water, other environmental media and biological materials. Sample preparation techniques such as preconcentration have to be often used to concentrate the target analytes. This treatment can significantly lower the limit of detection using conventional analytical procedures such as chromatography or spectrophotometry.

Several sample preparation procedures can be used in analytical chemistry. One of them is a well-known liquid-liquid extraction, which is based on the analyte partitioning between water and an immiscible organic solvent. However, currently alternative extraction methods known as solid phase extraction (SPE) are preferentially employed. During the extraction process, an aqueous sample passes through a solid phase (adsorbent) and afterwards is extracted by a suitable eluant (often organic solvent). In the most often applied SPE design the adsorbent is placed in a polypropylene cartridge (Buszewski \& Szultka 2012).

New forms of solid phase extraction have been developed in recent years. Very important ones are based on the use of magnetically responsive materials. The following text will be focused on the description of Magnetic solid phase extraction and Magnetic textile solid phase extraction.

\section{MAGNETIC SOLID PHASE EXTRACTION}

In order to extract target analytes from large sample volumes or difficult-to-handle samples, magnetic solid phase extraction (MSPE) employing magnetically responsive adsorbents was developed by Safarikova and Safarik in 1999. This approach has several advantages over traditional solid phase extraction performed in cartridges, namely: (a) it provides a rapid and simple analyte separation that avoids the need for centrifugation or filtration steps, (b) it avoids time-consuming and tedious on-column SPE procedures, (c) MSPE can also be used for samples containing suspended solids, (d) the magnetically responsive adsorbents may have high selectivity, even when complex biological and environmental samples are analyzed, (e) minimal amount of organic solvents for elution is necessary, (f) since the majority of sample impurities are diamagnetic, they do not interfere with magnetic particles during the magnetic separation step, and (g) automation of the whole process is possible, which leads to rapid, selective, sensitive, and repeatable methods for routine analyte determinations (Safarik \& Safarikova 2012; Azzouz et al. 2018). The scheme of MSPE is shown in Figure 1.

MSPE is based on the use of various magnetically responsive nano- and micromaterials, including iron oxides magnetite $\left(\mathrm{Fe}_{3} \mathrm{O}_{4}\right)$ and maghemite $\left(\gamma-\mathrm{Fe}_{2} \mathrm{O}_{3}\right)$, various types of ferrites $\left(\mathrm{MFe}_{2} \mathrm{O}_{4}\right)$ where $\mathrm{M}$ can be copper, nickel, manganese, magnesium, etc., pure ferromagnetic metals (iron, cobalt and nickel), and various metal alloys (FePt, CoPt). Both magnetite, maghemite and magnetic mixed iron oxides exhibit sufficient magnetic moment, good stability, and low toxicity and that's why they are predominantly used. They can be prepared using several simple precipitation or alternative procedures. Magnetic materials can serve as supports for the immobilization of an 
appropriate affinity ligand, or as magnetic labels for conversion of originally diamagnetic materials into magnetically modified ones.

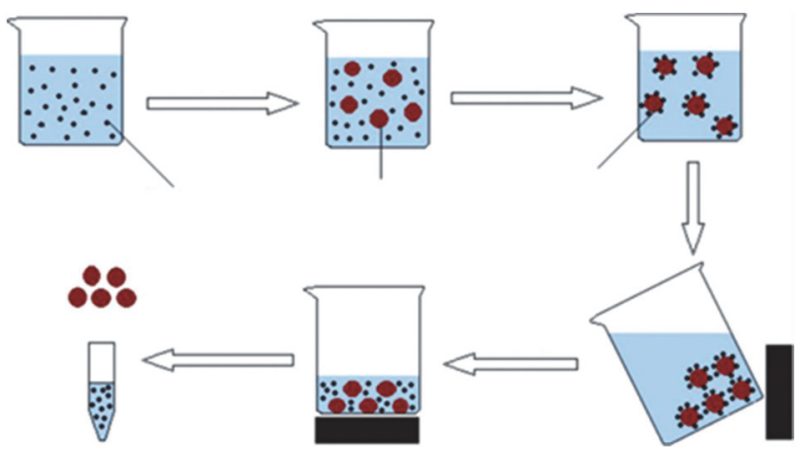

Figure 1. Magnetic solid phase extraction (reproduced from Chen et al. 2011)

Different types of materials have been coated onto the surfaces of magnetic particles. For example, graphene and graphene oxide, single wall and multi wall carbon nanotubes, metal-organic framework, molecularly imprinted polymers, ionic liquids, specific polymers or low molecular weight affinity ligands have been bound to magnetic particles and subsequently used as sorbents to extract and preconcentrate polyaromatic hydrocarbons, chlorophenols, phthalate esters, pesticides, fluoroquinolones, organochlorine pesticides, fungicides, hormones, monocyclic aromatic amines, bisphenol A, organophosphorus pesticides and other analytes from water solutions. The adsorption efficiency and selectivity of magnetic particles can be tuned by surface modifications with various composites and ligands, allowing the mechanisms for extracting trace-level analytes to match the nature of the target molecules. This technique greatly simplifies the extraction procedure and enhances the extraction efficiency. Magnetic adsorbents can be prepared in different shape, size and configuration. Magnetic separation is not influenced by the change of $\mathrm{pH}$, ionic strength or the presence of contaminating diamagnetic materials (AndradeEiroa et al. 2016; Hemmati et al. 2018; Azzouz et al. 2018).

MSPE is now a very useful and widely used preconcentration procedure for both organic and inorganic species. Currently, there are more than one thousand records in Web of Science and ca 1100 records in Scopus. Detailed information about the recent applications of MSPE in various disciplines can be found in variety of review papers (Ibarra et al. 2015; Ibrahim et al. 2015; Speltini et al. 2016; Vasconcelos \& Fernande 2017; Hemmati et al. 2018).

Specific problems during MSPE can appear when the sample volume is too big and very small magnetic particles are used. In this case, magnetic separation can take a long time and some loss of magnetic adsorbent can be observed. Due to these facts a complementary procedure has been developed recently, which is based on the use of magnetically responsive pieces of textile.

\section{MAGNETIC TEXTILE SOLID PHASE EXTRACTION}

A new type of low-cost preconcentration method, based on the use of magnetically modified textile (Magnetic textile solid phase extraction; MTSPE) has been developed recently (Safarik et al. 2018). In this procedure, a piece of an appropriate textile (fabric) is used as a carrier for the immobilization of a specific affinity, ion exchange or hydrophobic ligand. In order to prepare magnetically responsive adsorbent, an iron-based standard staple was inserted in the textile material using an office stapler (see Figure 2). Magnetically modified pieces of textile can be moved using appropriate laboratory magnetic stirrers in a similar way as magnetic stirring bars. At the end of the extraction process, the piece of textile can be easily and rapidly separated magnetically, as shown in Figure 3. The whole process of magnetic textile solid phase extraction is shown in Figure 4.

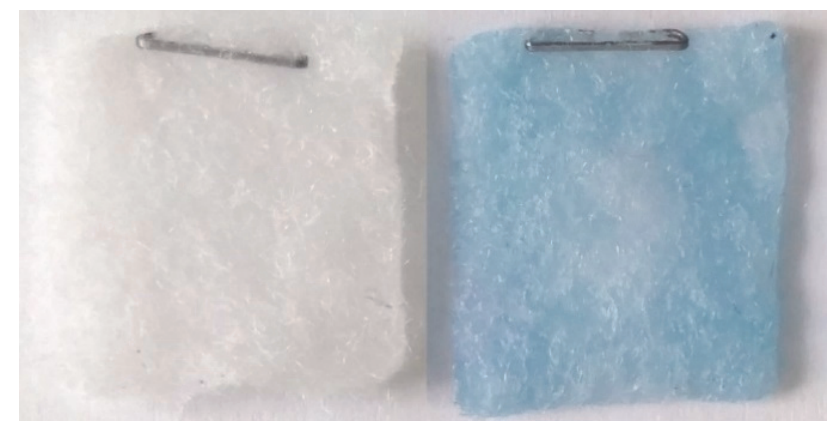

Figure 2. Magnetically responsive chitosan modified textile square and magnetically responsive chitosan modified textile square with adsorbed indigo carmine (reproduced from Safarik et al. 2019a) 

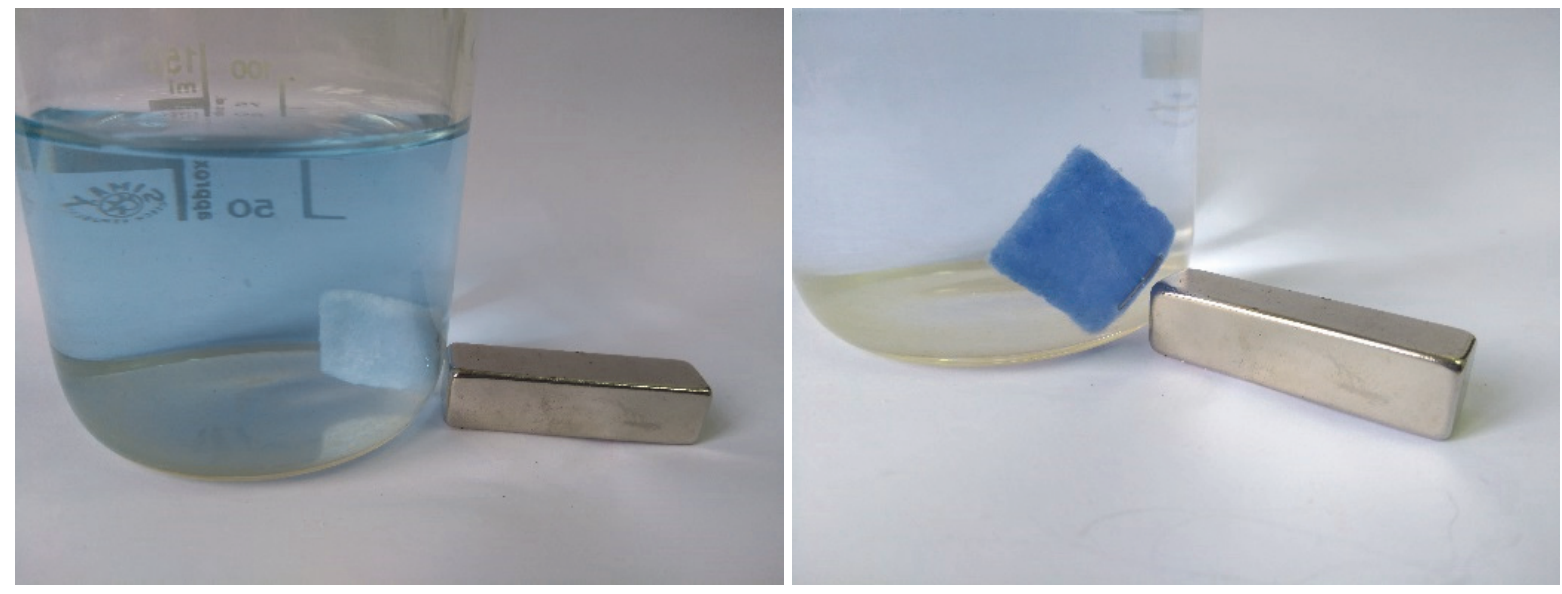

Figure 3. Magnetic separation of magnetically responsive textile squares

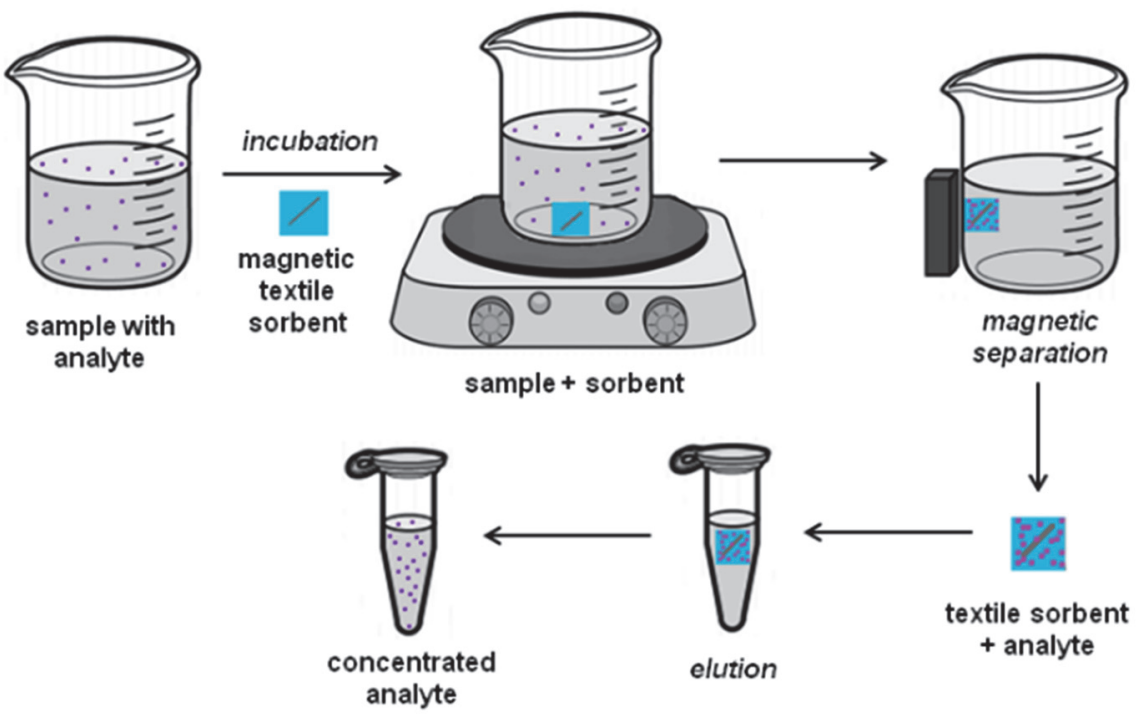

Figure 4. Magnetic solid phase extraction (reproduced from Safarik et al. 2018)

Various types of textile can be used for MTSPE. In the recent experiments, nonwoven acrylic felt was employed. The fibres have to be usually modified by an appropriate ligand. Reactive copper phthalocyanine dye covalently immobilized on the textile was used for the preconcentration of crystal violet and safranin $\mathrm{O}$ from diluted solutions (Safarik et al. 2018). Alternatively, textile fibres were modified with a positively charged polysaccharide chitosan (see Figure 5). Chitosan modified textile was applied for the preconcentration of food acid dyes tartrazine, azorubine and indigo carmine (Safarik et al. 2019; Safarik et al. 2019a) or blue fountain ink dye Acid blue 93 (Safarik \& Pospiskova 2018).

Similarly to standard solid phase separation procedures, analyte bound to magnetically responsive textile is usually eluted with a small amount of an appropriate eluant. After elution, standard analytical procedures including chromatography and spectrophotometry are used for analyte determination (Safarik et al. 2018; Safarik \& Pospiskova 2018; Safarik et al. 2019a).

A simple method enabling analysis of dyes adsorbed on magnetically modified textile, without the need of their elution, has been developed recently. After the dye preconcentration, photos of textile squares with the adsorbed dye were taken using a mobile phone or a digital camera (see Figure 6). Using an appropriate software, the square or rectangle covering maximum of homogeneously colored textile (without the staple) was cropped from the original image for the subsequent evaluation using ImageJ. Every image was analyzed using Color Inspector 3D plugin which is an integral part of ImageJ software. In this plugin, Median Cut option was used to reduce original range of colors into a single one "median" color. After that the HSB color space (Cheng et al. 2001) was applied to obtain three image parameters, namely hue (H), saturation (S) and brightness (B) (see Figure 7). The values of saturation were measured, which were proportional to the dye concentration in the analyzed samples (see Figure 8). Using this inexpensive, elution free assay it is possible to analyze dyes concentration in various solutions. 


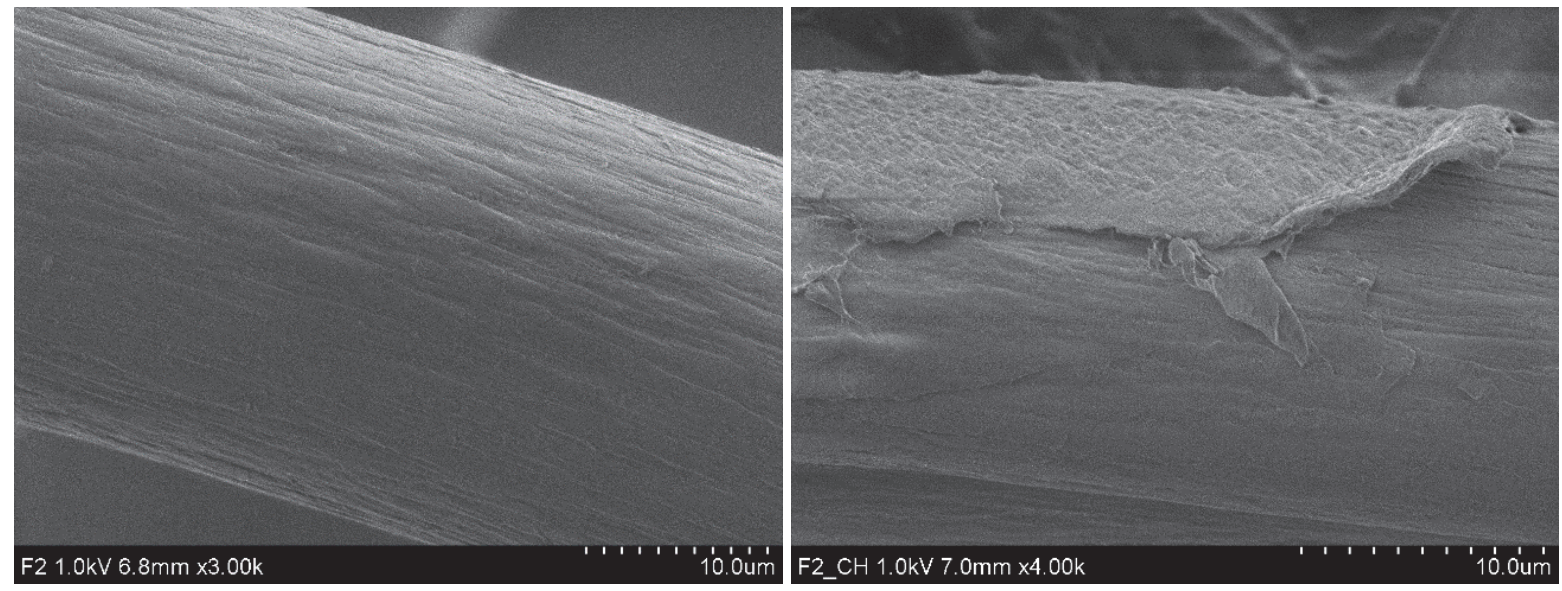

Figure 5. SEM image of native (left) and chitosan modified (right) acrylic fibers present in the non-woven textile used for MTSPE (reproduced from Safarik et al. 2019)

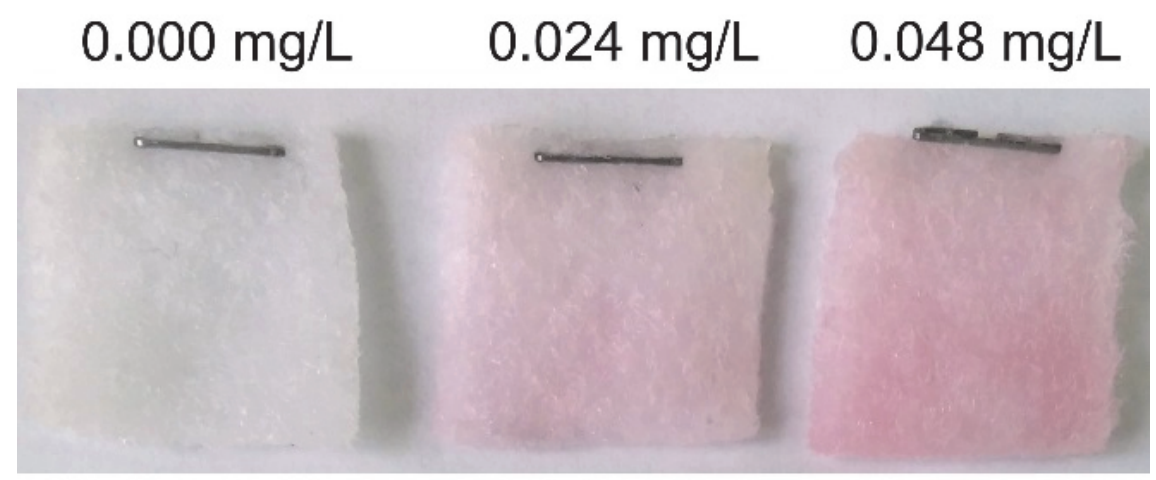
$0.072 \mathrm{mg} / \mathrm{L}$
$0.096 \mathrm{mg} / \mathrm{L}$
$0.120 \mathrm{mg} / \mathrm{L}$

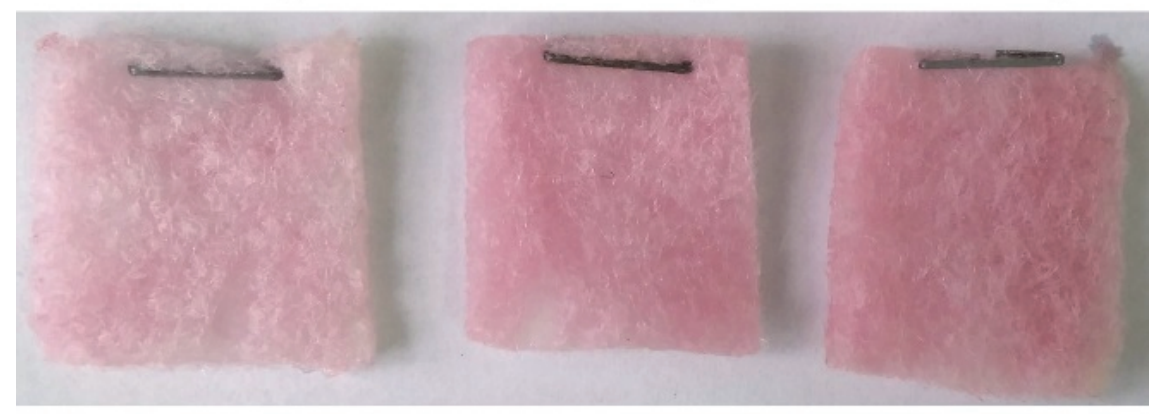

\section{$0.144 \mathrm{mg} / \mathrm{L} \quad 0.960 \mathrm{mg} / \mathrm{L}$}

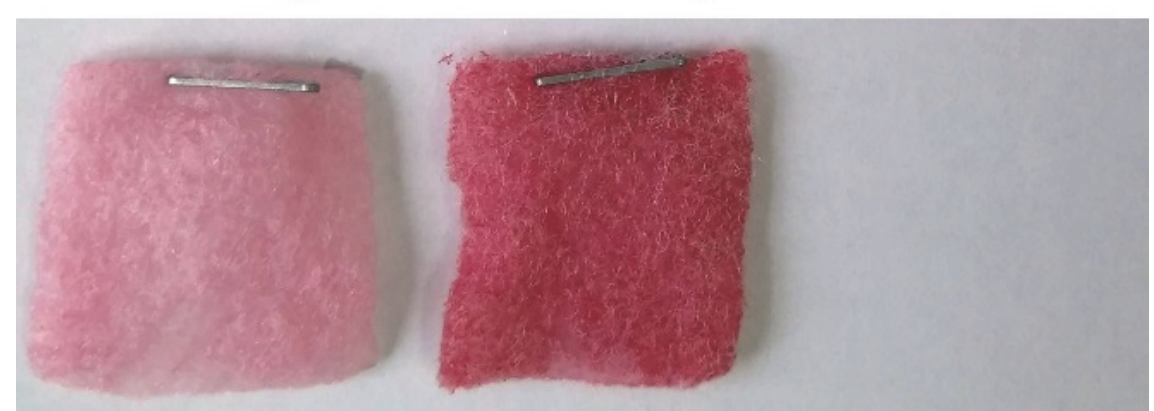

Figure 6. Examples of magnetically responsive, chitosan modified textile squares after azorubine extraction from water solution (100 mL; concentrations of the original solutions are shown)

(reproduced from Safarik et al. 2019) 


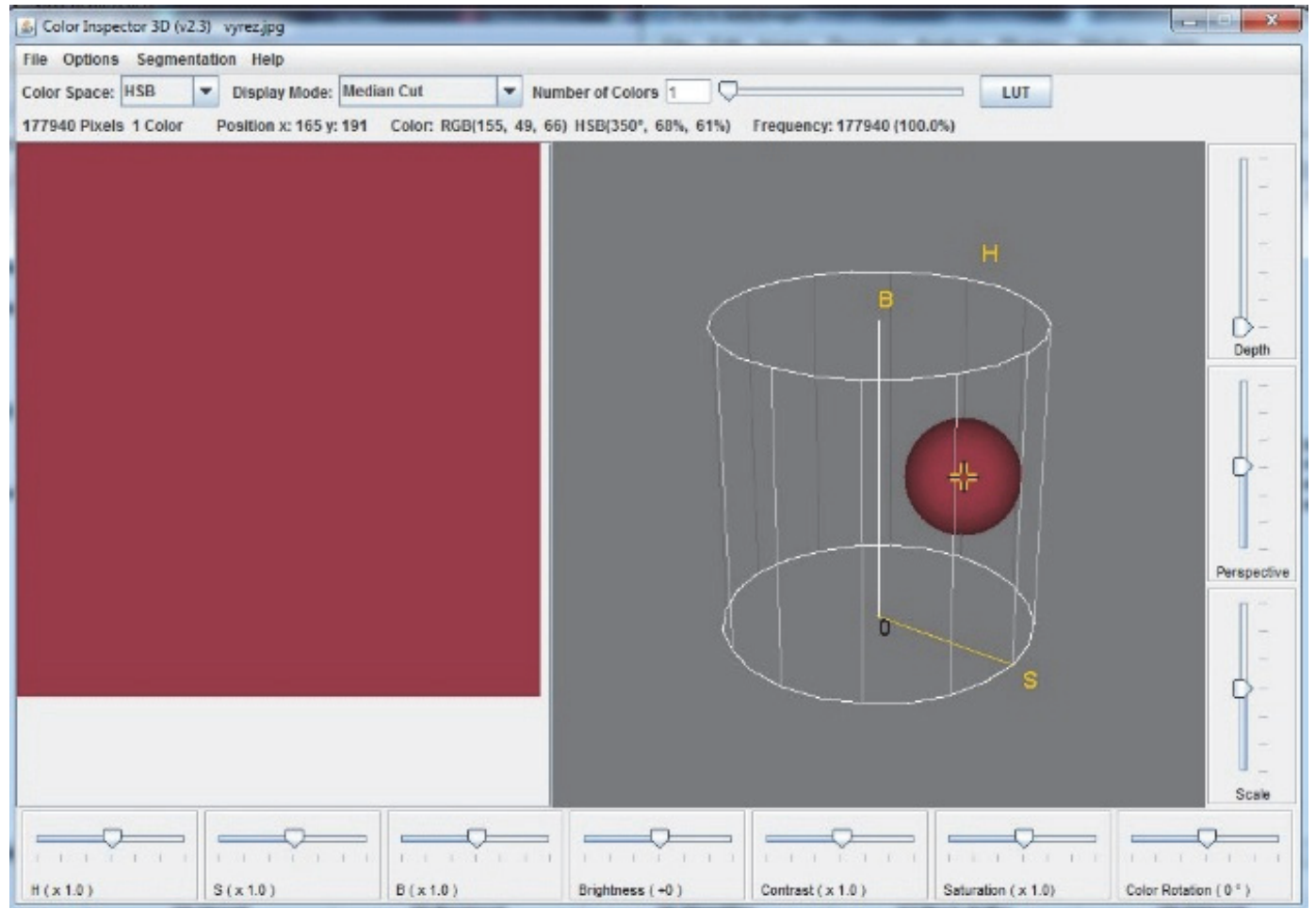

Figure 7. Appearance of the computer screen showing ImageJ software analyzing image of a textile square after MTSPE of azorubine. Reproduced from Safarik et al. 2019.

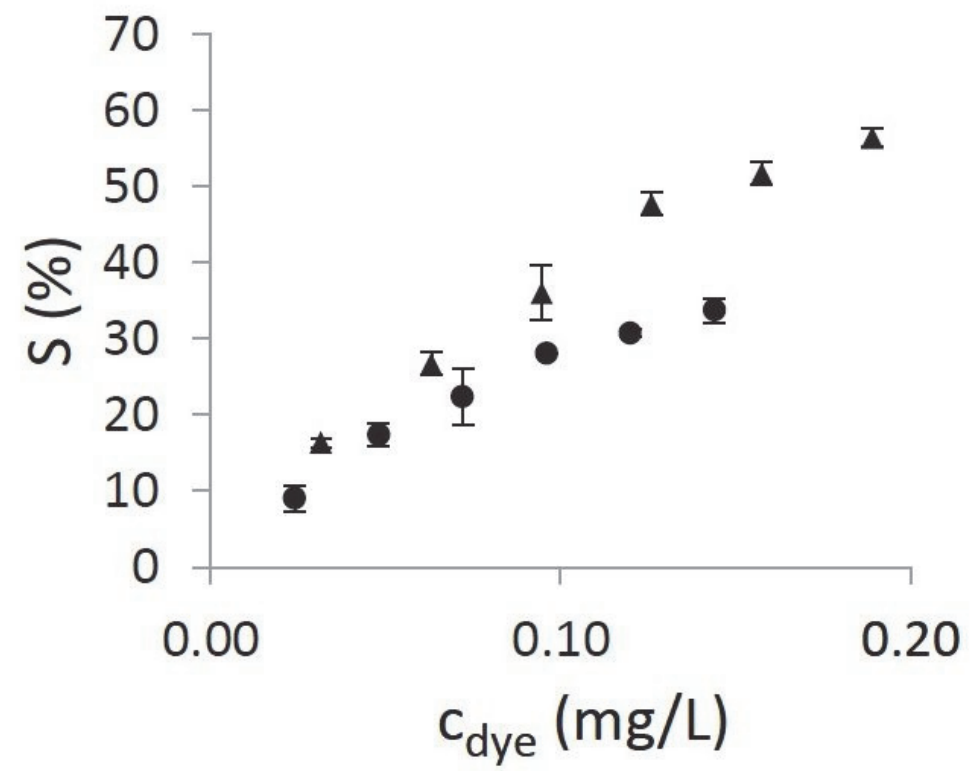

Figure 8. Dependence of values of saturation $(S)$ on the concentration of tartrazine $(\mathbf{\Delta})$ and azorubine ( $\bullet$ ). Reproduced from Safarik et al. 2019.

\section{CONCLUSIONS}

Magnetically responsive materials have already shown their great potential in analytical chemistry. In addition to already well established Magnetic solid phase extraction, a complementary procedure called Magnetic textile solid phase extraction has been developed recently. Both techniques can significantly lower the limit of detection of target analytes using conventional analytical procedures such as chromatography or spectrophotometry. Using MTSPE both elution and elution free assays can be performed. It has been proved that elution free assay enables inexpensive assay requiring only a digital camera or a mobile phone and a computer, in addition to freely available software. 


\section{ACKNOWLEDGMENTS}

This research was supported by the ERDF/ESF project "New Composite Materials for Environmental Applications" (No. CZ.02.1.01/0.0/0.0/17_048/0007399) and ERDF project "Development of pre-applied research in nanotechnology and biotechnology" (No. CZ.02.1.01/0.0/0.0/17_048/0007323). This work was carried out in the frame of the COST Actions CA16215 (European network for the promotion of portable, affordable and simple analytical platforms) and CA17107 (European Network to connect research and innovation efforts on advanced Smart Textiles).

\section{REFERENCES}

Andrade-Eiroa A, Canle M, Leroy-Cancellieri V, Cerdà V (2016) Solid-phase extraction of organic compounds: A critical review (Part I). TrAC Trends Anal Chem 80: 641-654

Azzouz A, Kailasa SK, Lee, SS, Rascón AJ, Ballesteros E, Zhang M, Kim K-H (2018) Review of nanomaterials as sorbents in solid-phase extraction for environmental samples. TrAC Trends Anal Chem 108: 347-369

Buszewski B, Szultka M (2012) Past, present, and future of solid phase extraction: A Review. Crit Rev Anal Chem 42: 198-213

Chen L, Wang T, Tong J (2011) Application of derivatized magnetic materials to the separation and the preconcentration of pollutants in water samples. TrAC Trends Anal Chem 30 (7): 1095-110

Cheng HD, Jiang XH, Sun Y, Wang J (2001) Color image segmentation: advances and prospects. Pattern Recognition 34 (12): 2259-2281

Hemmati M, Rajabi M, Asghari A (2018) Magnetic nanoparticle based solid-phase extraction of heavy metal ions: A review on recent advances. Microchim Acta 185: Article160

Ibarra IS, Rodriguez JA, Galan-Vidal CA, Cepeda A, Miranda JM (2015) Magnetic solid phase extraction applied to food analysis. J Chem 2015: Article No. 919414

Ibrahim WAW, Nodeh HR, Aboul-Enein, HY, Sanagi MM (2015) Magnetic solid-phase extraction based on modified ferum oxides for enrichment, preconcentration, and isolation of pesticides and selected pollutants. Crit Rev Anal Chem 45 (3): 270-287

Safarik I, Pospiskova K (2018) A simple extraction of blue fountain ink dye (Acid blue 93) from water solutions using Magnetic Textile Solid-Phase Extraction. Sep Sci plus 1: 48-51

Safarik I, Safarikova M (2012) Magnetic nanoparticles for in vitro biological and medical applications: An overview. In: Thanh NTK (ed) Magnetic Nanoparticles: From Fabrication to Biomedical and Clinical Applications. CRC Press/Taylor and Francis, 215-242

Safarik I, Baldikova E, Safarikova M, Pospiskova K (2018) Magnetically responsive textile for a new preconcentration procedure: Magnetic textile solid phase extraction. J Ind Text 48 (4): 761-771

Safarik I, Mullerova S, Pospiskova K (2019) Semiquantitative determination of food acid dyes by magnetic textile solid phase extraction followed by image analysis. Food Chem 274: 215-219

Safarik I, Mullerova S, Pospiskova K (2019a) Magnetically responsive textile for preconcentration of acid food dyes. Mater Chem Phys 232: 205-208

Safarikova M, Safarik I (1999) Magnetic solid phase extraction. J Magn Magn Mater 194: 108-112

Speltini A, Sturini M, Maraschi F, Profumo A (2016) Recent trends in the application of the newest carbonaceous materials for magnetic solid-phase extraction of environmental pollutants. Trends Environ Anal Chem 10: 11-23

Vasconcelos I, Fernandes C (2017) Magnetic solid phase extraction for determination of drugs in biological matrices. TRAC-Trends Anal Chem 89: 41-52 